\title{
MODULES WITH FINITELY MANY SUBMODULES
}

\author{
Gabriel Picavet and Martine Picavet-L'Hermitte \\ Received: 2 July 2015; Revised: 8 August 2015 \\ Communicated by Sait Halıcıoğlu
}

\begin{abstract}
We characterize ring extensions $R \subset S$ having FCP (FIP), where $S$ is the idealization of some $R$-module. As a by-product we exhibit characterizations of the modules that have finitely many submodules. Our tools are minimal ring morphisms, while Artinian conditions on rings are ubiquitous.
\end{abstract}

Mathematics Subject Classification (2010): 13B02, 13E10, 13B30, 13F10

Keywords: Idealization, $\Delta_{0}$-extension, SPIR, minimal ring extension, ramified, subintegral extension, FIP, FCP extension, Artinian ring

\section{Introduction and notation}

All rings $R$ considered are commutative, nonzero and unital; all morphisms of rings are unital. Let $R \subseteq S$ be a (ring) extension. The set of all $R$-subalgebras of $S$ is denoted by $[R, S]$. The extension $R \subseteq S$ is said to have FIP (for the "finitely many intermediate algebras property") if $[R, S]$ is finite. A chain of $R$-subalgebras of $S$ is a set of elements of $[R, S]$ that are pairwise comparable with respect to inclusion. We say that the extension $R \subseteq S$ has FCP (for the "finite chain property") if each chain of $R$-subalgebras of $S$ is finite. It is clear that each extension that satisfies FIP must also satisfy FCP. If the extension $R \subseteq S$ has FIP (FCP), we will sometimes say that $R \subseteq S$ is an FIP (FCP) extension. Our main tool are the minimal (ring) extensions, a concept introduced by Ferrand-Olivier [10]. Recall that an extension $R \subset S$ is called minimal if $[R, S]=\{R, S\}$. The key connection between the above ideas is that if $R \subseteq S$ has FCP, then any maximal (necessarily finite) chain $R=R_{0} \subset R_{1} \subset \cdots \subset R_{n-1} \subset R_{n}=S$, of $R$-subalgebras of $S$, with length $n<\infty$, results from juxtaposing $n$ minimal extensions $R_{i} \subset R_{i+1}, 0 \leq i \leq n-1$. The length of $[R, S]$, denoted by $\ell[R, S]$, is the supremum of the lengths of chains of $R$-subalgebras of $S$. In particular, if $\ell[R, S]=r$, for some integer $r$, there exists a maximal chain $R=R_{0} \subset R_{1} \subset \cdots \subset R_{r-1} \subset R_{r}=S$ of $R$-subalgebras of $S$ with length $r$. Against the general trend, we characterized arbitrary FCP and FIP extensions in [8], a joint paper by D. E. Dobbs and ourselves whereas most of papers on the subject are concerned with extensions of integral domains. Note that 
other papers by D. E. Dobbs [6], and D. E. Dobbs with P.-J. Cahen, T. G. Lucas [5], J. Shapiro [9], B. Mullins and ourselves [7] also went against the same trend. It is worth noticing here that FCP extensions of integral domains are (ignoring fields) extensions of overrings as a quick look at [5, Theorems 4.1,4.4] shows because FCP extensions are composites of finitely many minimal extensions.

The seminal work on FIP and FCP by R. Gilmer is settled for $R$-subalgebras of $K$ (also called overrings of $R$ ), where $R$ is a domain and $K$ its quotient field. In particular, [12, Theorem 2.14] shows that $R \subseteq S$ has FCP for each overring $S$ of $R$ only if $R / C$ is an Artinian ring, where $C=(R: \bar{R})$ is the conductor of $R$ in its integral closure. This necessary Artinian condition is not surprisingly present in all our results.

This paper is concerned with $R$-modules $M$ over a ring $R$ and ring extensions $R \subseteq R(+) M$, where $R(+) M$ is the idealization of $M$. The main results are as follows. Proposition 2.2 shows that $R \subseteq R(+) M$ has FCP if and only if the length of the $R$-module $M$ is finite, while Proposition 2.4 says that $R \subseteq R(+) M$ has FIP if and only if $M$ has finitely many $R$-submodules. This leads us to characterize $R$-modules having finitely many $R$-submodules in Corollary 2.7. An $R$-module $M$, with $C:=(0: M)$, has finitely many submodules if and only if the three following conditions are satisfied: $M$ is finitely generated, $R / C$ has finitely many ideals and $M_{P}$ is cyclic for any prime ideal $P$ of $R$ containing $C$ such that $R / P$ is infinite. Then Theorem 2.13 gives a structure theorem for these modules that are faithful.

Let $R$ be a ring. As usual, $\operatorname{Spec}(R)(\operatorname{resp} . \operatorname{Max}(R))$ denotes the set of all prime ideals (resp. maximal ideals) of $R$. If $I$ is an ideal of $R$, we set $\mathrm{V}_{R}(I):=\{P \in$ $\operatorname{Spec}(R) \mid I \subseteq P\}$. If $R \subseteq S$ is a ring extension and $P \in \operatorname{Spec}(R)$, then $S_{P}$ is the localization $S_{R \backslash P}$ and $(R: S)$ is the conductor of $R \subseteq S$. If $E$ is an $R$-module, $\mathrm{L}_{R}(E)$ is its length. We will shorten finitely generated module to f.g. module. Recall that a special principal ideal ring (SPIR) is a principal ideal ring $R$ with a unique nonzero prime ideal $M=R t$, such that $M$ is nilpotent of index $p>0$. Hence a SPIR is not a field. Each nonzero element of a SPIR is of the form $u t^{k}$ for some unit $u$ and some unique integer $k<p$. Finally, as usual, $\subset$ denotes proper inclusion and $|X|$ denotes the cardinality of a set $X$.

There are four types of minimal extension, but we only need ramified minimal extensions.

Theorem 1.1. [10, Théorème 2.2], [16, Theorem 3.3] Let $R \subset T$ be a ring extension and $M:=(R: T)$. Then $R \subset T$ is a ramified minimal extension if and only if $M \in \operatorname{Max}(R)$ and there exists $M^{\prime} \in \operatorname{Max}(T)$ such that $M^{\prime 2} \subseteq M \subset M^{\prime},[T / M$ : 
$R / M]=2\left(\right.$ resp. $\left.\mathrm{L}_{R}\left(M^{\prime} / M\right)=1\right)$, and the natural map $R / M \rightarrow T / M^{\prime}$ is an isomorphism.

Definition 1.2. An integral extension $f: R \hookrightarrow S$ is termed subintegral if all its residual extensions are isomorphisms and ${ }^{a} f$ is bijective [18].

A minimal morphism is ramified if and only if it is subintegral.

According to J. A. Huckaba and I. J. Papick [14], an extension $R \subseteq S$ is termed a $\Delta_{0}$-extension provided each $R$-submodule of $S$ containing $R$ is an element of $[R, S]$. We recall here for later use an unpublished result of the Gilbert's dissertation.

Proposition 1.3. [11, Proposition 4.12] Let $R \subseteq S$ be a ring extension with conductor $I$ and such that $S=R+R$ for some $t \in S$. Then the $R$-modules $R / I$ and $S / R$ are isomorphic. Moreover, each of the $R$-modules between $R$ and $S$ is a ring (and so there is a bijection from $[R, S]$ to the set of ideals of $R / I$ ).

We will use the following result. If $R_{1}, \ldots, R_{n}$ are finitely many rings, the ring $R_{1} \times \cdots \times R_{n}$ localized at the prime ideal $P_{1} \times R_{2} \times \cdots \times R_{n}$ is isomorphic to $\left(R_{1}\right)_{P_{1}}$ for $P_{1} \in \operatorname{Spec}\left(R_{1}\right)$. This rule works for any prime ideal of the product.

Rings which have finitely many ideals are characterized by D. D. Anderson and S. Chun [1], a result that will be often used.

Proposition 1.4. [1, Corollary 2.4] A commutative ring $R$ has only finitely many ideals if and only if $R$ is a finite direct product of finite local rings, SPIRs, and fields, and these are the localizations of $R$ at its maximal ideals.

Note that if $(R, M)$ is a local Artinian ring, then $R$ is finite if and only if $R / M$ is finite, since $M^{n}=0$ for some integer $n$. If $(R, M)$ is an Artinian local ring, we denote by $n(R)$ the nilpotency index of $M$.

From now on, a ring $R$ with finitely many ideals is termed an FMIR.

\section{Idealizations which are FCP or FIP extensions}

Let $M$ be an $R$-module. We consider the ring extension $R \subseteq R(+) M$, where $R(+) M$ is the idealization of $M$ in $R$.

Recall that $R(+) M:=\{(r, m) \mid(r, m) \in R \times M\}$ is a commutative ring whose operations are defined as follows:

$$
(r, m)+(s, n)=(r+s, m+n) \quad \text { and } \quad(r, m)(s, n)=(r s, r n+s m)
$$

Then $(1,0)$ is the unit of $R(+) M$, and $R \subseteq R(+) M$ is a ring morphism defining $R(+) M$ as an $R$-module, so that we can identify any $r \in R$ with $(r, 0)$. The following lemma will be useful for all this section. 
Lemma 2.1. Let $M$ be an $R$-module, then $R \subseteq R(+) M$ is a subintegral extension with conductor $(0: M)$.

Proof. If $(r, m) \in R(+) M$, then $(r, m)^{2}=2 r(r, m)-r^{2}(1,0)$ shows that $R(+) M$ is integral over $R$. Moreover, by [13, Theorem 25.1(3)], $\operatorname{Spec}(R(+) M)=\{P(+) M \mid$ $P \in \operatorname{Spec}(R)\}$ implies that $R \subseteq R(+) M$ is subintegral.

Set $S:=R(+) M$ and let $x \in(R: S)$. Then, we have $(x, 0)(0, m)=(0, x m) \in$ $R$ for any $m \in M$, so that $x \in(0: M)$. Conversely, any $x \in(0: M)$ gives $x(r, m)=(x r, 0) \in R$ for any $(r, m) \in R(+) M$, which implies $x \in(R: S)$. So, we get $(R: S)=(0: M)$.

Proposition 2.2. Let $M$ be an $R$-module, then $R \subseteq R(+) M$ has FCP if and only if $\mathrm{L}_{R}(M)<\infty$ and, if and only if $R /(0: M)$ is Artinian and $M$ is f.g. over $R$.

Proof. Set $S:=R(+) M$. Since $R \subseteq S$ is integral, $R \subseteq S$ has FCP if and only if $\mathrm{L}_{R}(S / R)<\infty$ by [8, Theorem 4.2]. By the same reference, this condition is equivalent to $R /(0: M) \cong R /(R: S)$ is Artinian and $R \subseteq S$ is module finite. Finally, note that $S / R \cong M$; and that $S$ is f.g over $R$ if (and only if) $S / R$ is f.g. over $R$.

For a submodule $N$ of an $R$-module $M$, we denote by $\llbracket N, M \rrbracket$ the set of all submodules of $M$ containing $N$ and set $\llbracket M \rrbracket:=\llbracket 0, M \rrbracket$. Recall that $M$ is called uniserial if $\llbracket M \rrbracket$ is linearly ordered.

Proposition 2.3. (Dobbs) Let $M$ be an $R$-module, then $R \subseteq R(+) M$ is a $\Delta_{0^{-}}$ extension because $[R, R(+) M]=\{R(+) N \mid N \in \llbracket M \rrbracket\}$.

Proof. The equality $[R, R(+) M]=\{R(+) N \mid N \in \llbracket M \rrbracket\}$ was proved by D. E. Dobbs in [6, Remark 2.9] using the bijection $\llbracket M \rrbracket \rightarrow[R, R(+) M], N \mapsto R(+) N$.

We say that an $R$-module $M$ is an FMS module if $M$ has finitely many $R$ submodules. An FMS $R$-module $M$ is Noetherian and Artinian and $R /(0: M)$ is a Noetherian and Artinian ring. We denote by $\nu_{R}(M)$ (or $\nu(M)$ ) the number of submodules of an FMS $R$-module $M$. Hence, $\nu(R)$ is the number of ideals of an FMIR $R$.

Proposition 2.4. Let $M$ be an $R$-module, then $R \subseteq R(+) M$ has FIP if and only if $M$ is an FMS module. In this case, $|[R, R(+) M]|=\nu(M)$.

Proof. Set $S:=R(+) M$. By Proposition 2.3, it follows that $R \subseteq S$ has FIP if and only if $M$ is an FMS module. In this case, $|[R, R(+) M]|=\nu(M)$.

We now intend to characterize FMS modules by using the previous proposition. 
Theorem 2.5. An $R$-module $M$ over a quasi-local ring $(R, P)$ is an FMS module if and only if the next conditions (1) and (2) hold with $C:=(0: M)$ :

(1) $M$ is finitely generated, and cyclic when $|R / P|=\infty$.

(2) $R / C$ is an FMIR.

If $M$ is an FMS R-module, $(R, P)$ is quasi-local, $|R / P|=\infty$, and $M=$ Re for some $e \in M$, then $M$ is uniserial, $\llbracket M \rrbracket=\left\{P^{j} e \mid j=0, \ldots, m\right\}$, with $m:=$ $n(R / C)=\nu(R / C)-1$ and $|[R, R(+) M]|=m+1$.

Assume in addition that $P=(0: M)$ and $|R / P|=\infty$. Then $R \subseteq R(+) M$ has FIP if and only if $M$ is simple, if and only if $R \subseteq R(+) M$ is minimal ramified.

Proof. Note that $R$-submodules and $R / C$-submodules of $M$ coincide.

Assume that $M$ is an FMS module. We first prove (1). Then Proposition 2.4 shows that $R \subseteq R(+) M$ has FIP, whence has FCP. We deduce from Proposition 2.2 that $M$ is f.g. and $(R / C, P / C)$ is local Artinian. Assume that $|R / P|=\infty$. Denote by $R e_{1}, \ldots, R e_{n}$, with $e_{i} \in M$, the finitely many cyclic submodules of $M$. Then for any $m \in M$, there is some $i$ such that $R m=R e_{i}$, so that $M=\cup_{i=1}^{n} R e_{i}$. We can then suppose that $M=\cup_{i=1}^{p} R f_{i}$, where $f_{i} \in\left\{e_{1}, \ldots, e_{n}\right\}$ and the $R f_{i}$ are incomparable. If $p=1$, then $M$ is cyclic. The case $p=2$ cannot happen because a group cannot be the union of two proper incomparable subgroups. We now show that $p>2$ leads to a contradiction. Let $\mathcal{F}$ be a(n infinite) set of representatives of the non-zero elements of $R / P$. Then, each $\alpha \in \mathcal{F}$ is a unit of $R$. For each $\alpha \in \mathcal{F}$, set $m_{\alpha}:=f_{1}+\alpha f_{2}$. Obviously $m_{\alpha} \notin R f_{1} \cup R f_{2}$. It follows that $m_{\alpha} \in R f_{i}$, for some $i \neq 1,2$. Let $\alpha, \beta \in \mathcal{F}, \alpha \neq \beta$. We claim that $m_{\alpha}$ and $m_{\beta}$ are not in the same $R f_{i}$. Deny, then $m_{\alpha}-m_{\beta}=(\alpha-\beta) f_{2} \in R f_{i}$ and $\alpha-\beta$ is a unit implies $f_{2} \in R f_{i}$, a contradiction. Therefore, $M$ is cyclic and (1) is proved.

To prove (2), we consider two cases. If $|R / P|<\infty$, then $|R / C|<\infty$ (see the remark after Proposition 1.4), so that $R / C$ is an FMIR.

Assume that $|R / P|=\infty$. It follows from (1) that $M=R e$ for some $e \in M$, so that $C=(0: e)$. Set $R^{\prime}:=R / C, P^{\prime}:=P / C$ and $I_{N}:=\left(N:_{R} e\right)$ for $N \in \llbracket M \rrbracket$. Then, $I_{N} \in \llbracket C, R \rrbracket$ and is such that $N=I_{N} e$. Conversely, $I \in \llbracket C, R \rrbracket$ is such that $I=I_{I e}$ with $I e \in \llbracket M \rrbracket$, since $C \subseteq I$. We define a preserving order bijective map $\psi: \llbracket C, R \rrbracket \rightarrow \llbracket M \rrbracket$ by $I \mapsto I e$. It follows that $R^{\prime}$ is an FMIR (either a field or a SPIR) and $\nu(M)=\nu(R / C)$. Then, (2) is proved.

Now, assume that (1) and (2) hold. There is no harm to suppose that $C=0$ and that $R$ is an FMIR, so that $(R, P)$ is local Artinian. If $|R / P|<\infty$, we get that $|M|<\infty$ and then $M$ is an FMS module. Assume that $|R / P|=\infty$, and that $M=R e$ is cyclic. The assertion is clear if $M=0$. Assume $M \neq 0$. If $P=0$, then 
$M$ is a one-dimensional vector space over the field $R$, so that $\nu(M)=2=\nu(R)$. If $P \neq 0$, consider $S:=R(+) M=R+R f$, where $f=(0, e)$. From Proposition 1.3 we deduce that $|\llbracket R, S \rrbracket|<\infty$, since $R$ is an FMIR and also that there are bijective maps $\llbracket R \rrbracket \rightarrow[R, S]$ and $\llbracket R, S \rrbracket \rightarrow \llbracket M \rrbracket$. In fact $\llbracket R, S \rrbracket=\{R(+) N \mid N \in \llbracket M \rrbracket\}$. By Proposition 2.3, $M$ is an FMS module.

Assume that $M$ is an FMS $R$-module, $(R, P)$ is quasi-local, $|R / P|=\infty$, and $M=R e$ for some $e \in M$. If $R^{\prime}$ is a SPIR, there is some $x \in P$, whose class $\bar{x} \in R^{\prime}$ is such that $P^{\prime}=R^{\prime} \bar{x}, \bar{x}^{m}=0$ and $\bar{x}^{m-1} \neq 0$, for $m:=n\left(R^{\prime}\right)>1$. It follows that $\llbracket C, R \rrbracket=\left\{P^{j}+C \mid j \in\{0, \ldots, m\}\right\}$ and $\llbracket M \rrbracket=\left\{P^{j} e \mid j \in\{0, \ldots, m\}\right\}$ (to see this, use the above bijection $\psi$ ). If $R^{\prime}$ is a field, then $P=C$ gives $m=1$. In both cases, $M$ is uniserial, $m:=n(R / C)=\nu(R / C)-1$ and $|[R, R(+) M]|=m+1$.

To end, assume that $(R, P)$ is quasi-local with $|R / P|=\infty$. Let $M$ be a simple $R$ module, with $P=(0: M)$. Then $[R, R(+) M]=\{R, R(+) M\}$ by Proposition 2.3. It follows that $R \subseteq R(+) M$ has FIP and is a minimal ramified extension since minimal subintegral. The converse is obvious.

Example 2.6. We give this example due to the referee showing that the condition $|R / P|=\infty$ in Theorem 2.5 is necessary in order to have $M$ a simple module when $M$ is an FMS module. Let $R$ be a finite field, and let $M:=R \bigoplus R$. Then, $R \subseteq R(+) M$ has FIP since $M$ has only finitely many submodules and $(0: M)=$ $\{0\}=P$, but $M$ is not a simple $R$-module.

Corollary 2.7. Let $M$ be an $R$-module and $C:=(0: M)$. Then $M$ is an FMS module if and only if the two following conditions hold:

(1) $M$ is f.g. and $M_{P}$ is cyclic over $R_{P}$ for all $P \in \mathrm{V}(C)$ such that $|R / P|=\infty$.

(2) $R / C$ is an FMIR.

In case $(1),(2)$ both hold, set $\left\{P_{1}, \ldots, P_{n}\right\}=\mathrm{V}(C)$ and suppose that each $\left|R / P_{i}\right|=$ $\infty$. Then, for each $i$, there exist some $e_{i} \in M$, such that $M_{P_{i}}=R_{P_{i}}\left(e_{i} / 1\right)$ and, $M$ is generated by the $e_{1}, \ldots, e_{n}$.

Proof. If $M$ is an FMS module, Proposition 2.4 shows that $R \subseteq R(+) M$ has FIP, and then has FCP. Hence, $M$ is f.g. and $R / C$ is Artinian by Proposition 2.2. Let $P \in \mathrm{V}(C)$, then $M_{P}$ is an FMS $R_{P}$-module, so that we can use Theorem 2.5. It follows that $R_{P} / C_{P} \cong(R / C)_{P}$ is an FMIR, and so is $R / C$, since $|\mathrm{V}(C)|<\infty$, which gives (2). Moreover, for $P \in \mathrm{V}(C)$ with $|R / P|=\infty$, Theorem 2.5 gives that $M_{P}$ is cyclic and (1) holds.

Conversely, if (1) and (2) hold, they also hold for each $M_{P}$, where $P \in \mathrm{V}(C)$. Theorem 2.5 gives that $M_{P}$ is an FMS module for any $P \in \mathrm{V}(C)$. To show that $M$ 
is an FMS module, there is no harm to suppose that $C=0$, so that $R$ is Artinian, with $\operatorname{Max}(R)=\left\{P_{1}, \ldots, P_{n}\right\}$. Now if $N$ is a submodule of $M$, it is well known that $N=\cap_{i=1}^{n} \varphi_{i}^{-1}\left(N_{P_{i}}\right)$, where $\varphi_{i}: M \rightarrow M_{P_{i}}$ is the natural map and thus $M$ is an FMS module.

Now, assume that (1) and (2) hold and that $|R / P|=\infty$ for any $P \in \mathrm{V}(C)=$ $\left\{P_{1}, \ldots, P_{n}\right\}$. For each $j=1, \ldots, n$, there is some $e_{j} \in M$ such that $M_{P_{j}}=$ $R_{P_{j}}\left(e_{j} / 1\right)$. Set $M^{\prime}:=R e_{1}+\cdots+R e_{n}$. It is easy to show that $M_{P_{j}}^{\prime}=M_{P_{j}}$ for $j=1, \ldots, n$. Observe that $\mathrm{V}(C)=\operatorname{Supp}(M)$, because $M$ is f.g. ([2, Proposition 17, ch. II, p.133]). Now let $P \in \operatorname{Max}(R) \backslash \mathrm{V}(C)$. We get that $M_{P}^{\prime} \subseteq M_{P}=0$ and then $M^{\prime}=M$.

Let $N$ be a submodule of an $R$-module $M$. By Proposition 2.3, $R(+) N$ is an $R$-subalgebra of $R(+) M$ and then $R(+) M$ is an $(R(+) N)$-algebra. Even if $R \subseteq R(+) M$ does not have FCP (resp. FIP), it may be that $R(+) N \subseteq R(+) M$ has FCP (resp. FIP).

Any $(R(+) N)$-subalgebra of $R(+) M$ is an $R$-subalgebra of $R(+) M$, and then is of the form $R(+) N^{\prime}$, for some $N^{\prime} \in \llbracket N, M \rrbracket$ since $R(+) N \subseteq R(+) N^{\prime}$. Conversely, for any $R$-subalgebra $N^{\prime}$ of $M$ containing $N, R(+) N^{\prime}$ is an $(R(+) N)$-subalgebra of $R(+) M$. In particular, $R(+) N \subseteq R(+) M$ is a minimal extension if and only if $M / N$ is a simple module.

Proposition 2.8. Let $N$ be a submodule of an $R$-module $M$. Then:

(1) $R(+) N \subseteq R(+) M$ is a $\Delta_{0}$-extension.

(2) $R(+) N \subseteq R(+) M$ has $F C P$ if and only if $\mathrm{L}_{R}(M / N)<\infty$. In this case, $\ell[R(+) N, R(+) M]=\mathrm{L}_{R}(M / N)$.

(3) $R(+) N \subseteq R(+) M$ has FIP if and only if $M / N$ is an FMS module. In this case, $|[R(+) N, R(+) M]|=\nu(M / N)$.

Proof. (1) By Proposition 2.3, $R \subseteq R(+) M$ is a $\Delta_{0}$-extension. Since an $(R(+) N)$ submodule $S$ of $R(+) M$ containing $R$ is also an $R$-submodule of $R(+) M$, we get that $S$ is a ring, so that $R(+) N \subseteq R(+) M$ is a $\Delta_{0}$-extension.

(2) By Lemma 2.1, $R \subseteq R(+) M$ is integral and so is $R(+) N \subseteq R(+) M$. Therefore, the following conditions are equivalent:

- $R(+) N \subseteq R(+) M$ has FCP

- there exists a finite chain of minimal finite extensions going from $R(+) N$ to $R(+) M([8$, Theorem $4.2(2)])$

- there is a finite maximal chain of $R$-submodules of $M$ going from $N$ to $M$

- $\mathrm{L}_{R}(M / N)<\infty$. 
In this case, $\ell[R(+) N, R(+) M]=\mathrm{L}_{R}(M / N)$, the supremum of the lengths of chains of submodules of $M$ containing $N$.

(3) The following conditions are equivalent:

- $R(+) N \subseteq R(+) M$ has FIP

- there are finitely many $(R(+) N)$-subalgebras of $R(+) M$

- there are finitely many $R$-subalgebras of $R(+) M$ containing $R(+) N$

- there are finitely many $R$-submodules of $M$ containing $N$

- $M / N$ is an FMS module.

In this case, $|[R(+) N, R(+) M]|$ is also the number of $R$-submodules of $M$ containing $N$, which is also $\nu(M / N)$.

We consider now the special case where $M$ is an ideal $I$ of $R$.

Proposition 2.9. Let $I$ be an ideal of a ring $R, S:=R(+) R$ and $T:=R(+) I$. Then:

(1) $R \subseteq S$ has FCP if and only if $\mathrm{L}_{R}(R)<\infty$ if and only if $R$ is Artinian. In this case, $\ell[R, R(+) R]=\mathrm{L}_{R}(R)$.

(2) $R \subseteq T$ has $F C P$ if and only if $\mathrm{L}_{R}(I)<\infty$ if and only if $I$ is finitely generated and $R /(0: I)$ is Artinian. In this case, $\ell[R, R(+) I]=\mathrm{L}_{R}(I)$.

(3) $R \subseteq S$ has FIP if and only if $R$ is an FMIR. In this case, $|[R, R(+) R]|=$ $\nu(R)$.

(4) $R \subseteq T$ has FIP if and only if $\llbracket I \rrbracket$ is finite. In this case, $|[R, R(+) I]|=\nu(I)$.

Proof. Propositions 2.2 and 2.8 with $M$ equal to $R$ or $I$ give most of the results because taking $N=0$ gives $R(+) 0 \cong R$.

Proposition 2.10. Any f.g. module over a ring $R$ is an FMS module if and only if $R$ is a finite ring.

Proof. If $R$ is finite, then $\llbracket M \rrbracket$ is finite for any f.g. $R$-module $M$. Conversely, let $R$ be a ring such that any f.g. $R$-module is an FMS module. Set $S:=$ $R[X, Y] /\left(X^{2}, X Y, Y^{2}\right)=R[x, y]$, where $x$ and $y$ are respectively the classes of $X$ and $Y$ in $S$. Then $S$ is an $R$-module with basis $\{1, x, y\}$. For each $\alpha \in R$, set $S_{\alpha}:=R(x+\alpha y)$, which is an $R$-submodule of $S$. If $\alpha, \beta \in R, \alpha \neq \beta$, then $S_{\alpha} \neq S_{\beta}$. Therefore, $|R|=\infty$ gives a contradiction and $R$ is a finite ring.

Remark 2.11. If $N$ is a submodule of an $R$-module $M$, Proposition 2.2 shows that $R \subseteq R(+) M$ has $F C P$ if and only if $R \subseteq R(+) N$ and $R \subseteq R(+)(M / N)$ have $F C P$. This property does not hold for FIP. It is enough to consider a 2-dimensional vector space $M$ over an infinite field, and a 1-dimensional subspace $N$ because $N$ and $M / N$ are FMS modules, while $M$ is not. 
Example 2.12. In the following examples, we mix properties of this section and [17, Section 3].

(1) Let $k$ be a field, $n>1$ an integer, $E$ an $n$-dimensional $k$-vector space with basis $\left\{e_{1}, \ldots, e_{n}\right\}$ and set $R:=k^{n}$. We can equip $E$ with the structure of an $R$ module by the following law: for $\left(a_{1}, \ldots, a_{n}\right) \in R$ and $x=\sum_{i=1}^{n} x_{i} e_{i}, x_{i} \in k$, we set $\left(a_{1}, \ldots, a_{n}\right) x:=\sum_{i=1}^{n} a_{i} x_{i} e_{i}$. Then $E$ is generated over $R$ by $\left\{e_{1}, \ldots, e_{n}\right\}$ and is faithful, while $R$ is an FMIR. Finally, the prime (maximal) ideals of $R$ are the ideals $P_{i}:=\left\{\left(a_{1}, \ldots, a_{n}\right) \in R \mid a_{i}=0\right\}$ for $i=1, \ldots, n$, so that $R_{P_{i}} \cong k$. The canonical base $\left\{\varepsilon_{1}, \ldots, \varepsilon_{n}\right\}$ of $R$ over $k$ is such that each $\varepsilon_{i} \notin P_{i}$. We have $\varepsilon_{i} e_{j}=0$ for each $i, j \in\{1, \ldots, n\}$ such that $i \neq j$, so that $e_{j} / 1=0$ in $R_{P_{i}}$ for $j \neq i$. It follows that $E_{P_{i}}=\sum_{j=1}^{n} R_{P_{i}}\left(e_{j} / 1\right)=R_{P_{i}}\left(e_{i} / 1\right)$ is cyclic over $R_{P_{i}} \cong k$. Then, whatever $|k|$ may be, Corollary 2.7 gives that $E$ is an FMS $R$-module. But, as soon as $|k|=\infty$ and $n \geq 2, E$ is infinite. Since $E_{P_{i}} \cong k\left(e_{i} / 1\right)$ is one-dimensional over $k$, $E_{P_{i}}$ has only two $R_{P_{i}}$-submodules. Set $F:=\prod_{i=1}^{n} E_{P_{i}}$ and consider the canonical injective morphism of $R$-modules $\varphi: E \rightarrow F$ and the projections $\varphi_{i}: F \rightarrow E_{P_{i}}$. Any $R$-submodule $N$ of $F$ is of the form $N^{\prime}:=\prod_{i=1}^{n} N_{i}$, where $N_{i}=\varphi_{i}(N)$, because $N \subseteq N^{\prime} \subseteq \sum_{i=1}^{n} \varepsilon_{i} N$. Now $\varphi$ is a $k$-isomorphism because $\operatorname{Dim}_{k}(E)=\operatorname{Dim}_{k}(F)$, whence an $R$-isomorphism. It follows that $\nu_{R}(E)=2^{n}$.

By Proposition 2.4, $k^{n} \subseteq k^{n}(+) E$ has FIP, and $k \subseteq k^{n}$ has FIP by [4, Proposition 3, p. 29] (another proof follows from [7, Theorem III.5]). But, always in view of Proposition 2.4, if $|k|=\infty$ and $n \geq 2$, then $k \subseteq k(+) E$ has not FIP, so that $k \subseteq k^{n}(+) E$ has not FIP.

(1') We keep the context of (1). Set $\mathcal{R}:=\prod_{i=1}^{n}\left(k /\left(0: e_{i}\right)\right)$. Since $\left(0: e_{i}\right)=0$ for each $i$, we get $\mathcal{R}=k^{n}$. Then $k \subset \mathcal{R}$ has FIP while $k \subseteq k(+) E$ has not FIP.

(2) Let $k$ be an infinite field, $n>1$ an integer and $E$ an $n$-dimensional vector space over $k$. Let $u \in \operatorname{End}(E)$ with minimal polynomial $X^{n}$. Then, $u^{n}=0$ and $u^{n-1}\left(e_{1}\right) \neq 0$ for some $e_{1} \in E$. If $e_{i}:=u^{i-1}\left(e_{1}\right)$ for any $i \in\{1, \ldots, n\}$, an easy induction shows that $\left\{e_{1}, \ldots, e_{n}\right\}$ is a basis of $E$ over $k$. Set $R:=k[u]$, then $E$ is a faithful $R$-module with scalar multiplication defined by $P(u) \cdot x:=P(u)(x)$, for $P(X) \in k[X]$ and $x \in E$. Since $R \cong k[X] /\left(X^{n}\right)$ is a SPIR and $E=R \cdot e_{1}$ because $e_{i}=u^{i-1} \cdot e_{1}$ for each $i$, then by Theorem 2.5, $E$ is an FMS $R$-module and $R \subseteq R(+) E$ has FIP by Proposition 2.4.

(2') Let $R$ be a ring, $n>1$ an integer and $I_{1}, \ldots, I_{n}$ ideals of $R$ distinct from $R$, but not necessarily distinct, such that $\cap_{j=1}^{n} I_{j}=0$. Such a family $\left\{I_{1}, \ldots, I_{n}\right\}$ of ideals of $R$ is called a separating family, a reference to Algebraic Geometry where a finite family of morphisms $\left\{f_{j}: M \rightarrow M_{j} \mid j=1, \ldots, n\right\}$ of $R$-modules is 
called separating if $\cap_{j=1}^{n}$ ker $f_{j}=0$. In [17, Section 3], we study the ring extension $R \subseteq \prod_{j=1}^{n}\left(R / I_{j}\right)=: \mathcal{R}$ associated to a separating family.

We keep the context of (2). Since $u^{n}=0, u^{n-1}\left(e_{1}\right) \neq 0$ and $e_{j}=u^{j-1}\left(e_{1}\right)$ for any $j \in\{1, \ldots, n\}$, a short calculation gives $I_{j}:=\left(0:_{R} e_{j}\right)=R u^{n-j+1}$. Then, $\cap_{j=1}^{n} I_{j}=0$ because $I_{1}=R u^{n}=0$ and $\left\{I_{1}, \ldots, I_{n}\right\}$ is a separating family such that $I_{j} \subset I_{j+1}$ for each $j \in\{1, \ldots, n-1\}$. Moreover, $R / I_{j}=R / R u^{n-j+1} \cong$ $k[X] /\left(X^{n-j+1}\right)$. Set $M:=R u, \mathcal{R}:=\prod_{i=1}^{n}\left(R /\left(0: e_{i}\right)\right)$ and $J_{j}:=\cap_{k=1, k \neq j}^{n} I_{k}$. Then, $J_{1}=I_{2} \cong\left(X^{n-1}\right) /\left(X^{n}\right)$ and $J_{j}=0$ for each $j>1$. Apply [17, Corollary 3.10]. We have $\sum_{j=1}^{n} J_{j}=I_{2}$, giving that $R / \sum_{j=1}^{n} J_{j}=R / I_{2} \cong k[X] /\left(X^{n-1}\right)$ is a SPIR and $|R / M|=\infty$, because $R / M \cong k$. Since $I_{1}+J_{1}=I_{2} \cong\left(X^{n-1}\right) /\left(X^{n}\right)$ and $I_{j}+J_{j}=I_{j} \cong\left(X^{n-j+1}\right) /\left(X^{n}\right)$ for each $j>1$, it is enough to take $n>3$ to get that $R \subset \mathcal{R}$ has not FIP.

(3) Let $M=\sum_{i=1}^{n} R e_{i}$ be a faithful Artinian $R$-module and set $\mathcal{R}:=\prod_{i=1}^{n}(R /(0$ : $\left.e_{i}\right)$ ). Since $M$ is faithful, we have $(0: M)=0$. Then, $R$ is an Artinian ring in view of [15, Theorem 2, page 180] because $M$ is a finitely generated Artinian module, and $R \subseteq R(+) M$ has FCP by Proposition 2.2. Since $(0: M)=\cap_{i=1}^{n}\left(0: e_{i}\right)=0$, the family $\left\{\left(0: e_{i}\right)\right\}_{i=1, \ldots, n}$ is separating and $R \subseteq \mathcal{R}$ has FCP by [17, Proposition $3.1]$.

Examples (1') and (2') show that for a finitely generated $R$-module $M=\sum_{i=1}^{n} R e_{i}$ such that $\left\{\left(0: e_{1}\right), \ldots,\left(0: e_{n}\right)\right\}$ is a separating family, we may have only one of the two extensions $R \subseteq R(+) M$ and $R \subseteq \prod_{i=1}^{n}\left(R /\left(0: e_{i}\right)\right)$ which has FIP, and not the other one.

(4) Let $k$ be an infinite field, $n>1$ an integer and $E$ an $n$-dimensional vector space over $k$. Let $u \in \operatorname{End}(E)$ with minimal polynomial $\pi_{u}(X):=\prod_{i=1}^{s} P_{i}^{\alpha_{i}}(X)$, with each $P_{i}(X) \in k[X]$ of degree $1, P_{i}(X) \neq P_{j}(X)$ for $i \neq j$, and such that $n=\sum_{i=1}^{s} \alpha_{i}$. For each $i$, set $E_{i}:=\operatorname{ker}\left(P_{i}^{\alpha_{i}}(u)\right)$. The "Lemme des noyaux" [4, Proposition 3, ch. VII, p. 30] gives that $E=\bigoplus_{i=1}^{s} E_{i}(*)$, with $\alpha_{i}=\operatorname{dim}_{k}\left(E_{i}\right)$. If $R:=k[u]$, then $E$ is a faithful $R$-module for the scalar multiplication defined by $P(u) \cdot x:=P(u)(x)$, for $P(X) \in k[X]$ and $x \in E$. Since $R \cong k[X] /\left(\pi_{u}(X)\right)$ is an Artinian FMIR, to conclude that $E$ is an FMS module over $R$ by applying Corollary 2.7, we need only to show that $E_{M}$ is cyclic for each $M \in \operatorname{Max}(R)=$ $\left\{M_{1}, \ldots, M_{s}\right\}$ where $M_{i}:=P_{i}(u) R$. We next prove that $E_{M_{i}} \cong\left(E_{i}\right)_{M_{i}}$ as $R_{M_{i}}{ }^{-}$ modules. Let $x \in E_{j}$ for some $j \neq i$, then $P_{j}^{\alpha_{j}}(u)(x)=0$ and $P_{j}^{\alpha_{j}}(u)$ is a unit in $R_{M_{i}}$ since $P_{j}(X) \notin\left(P_{i}(X)\right)$. It follows that $x / 1=0$ in $E_{M_{i}}$, so that $E_{M_{i}} \cong\left(E_{i}\right)_{M_{i}}$ by $(*)$. Now, we are reduced to $(2)$ with $P_{i}^{\alpha_{i}}(u)=0$ in $\left(E_{i}\right)_{M_{i}}$, so that each $\left(E_{i}\right)_{M_{i}}$ is cyclic over $R_{M_{i}}$ and Corollary 2.7 holds. 
Theorem 2.13. A faithful R-module $M$ is an FMS module if and only if the two following conditions are satisfied:

(1) $R$ is an FMIR which is a direct product of two rings $R^{\prime} \times R^{\prime \prime}$, where $\left|R^{\prime}\right|<\infty$ and $\left|R^{\prime \prime} / P\right|=\infty$ for any $P \in \operatorname{Spec}\left(R^{\prime \prime}\right)$.

(2) $M$ is the direct product of a finite $R^{\prime}$-module and a rank one projective $R^{\prime \prime}$-module.

Proof. If $M$ is an FMS module, $R$ is an FMIR and $M$ is f.g. over $R$ by Corollary 2.7. Then by Proposition 1.4, $R=\prod_{i=1}^{n} R_{i}$, a product of local rings that are either finite, or a SPIR, or a field. Let $R^{\prime}$ be the ring product of the $R_{i}$ that are finite and $R^{\prime \prime}$ the product of the others. Then $\left|R^{\prime}\right|<\infty$ and a SPIR factor $\left(R_{i}, P_{i}\right)$ of $R^{\prime \prime}$ is such that $\left|R_{i} / P_{i}\right|=\infty$ because $R_{i}$ is local Artinian. When $R_{i}$ is an infinite field, take $P_{i}=0$. So, (1) holds with $R=R^{\prime} \times R^{\prime \prime}$.

Set $M^{\prime}:=R^{\prime} M=\left\{\left(r^{\prime}, 0\right) m \mid r^{\prime} \in R^{\prime}, m \in M\right\}$ and $M^{\prime \prime}:=R^{\prime \prime} M=\left\{\left(0, r^{\prime \prime}\right) m \mid\right.$ $\left.r^{\prime \prime} \in R^{\prime \prime}, m \in M\right\}$. By [3, Remarque 3, ch.II, p.32], we get $M=M^{\prime} \bigoplus M^{\prime \prime} \cong M^{\prime} \times$ $M^{\prime \prime}, R^{\prime} M^{\prime \prime}=R^{\prime \prime} M^{\prime}=0$ and $\left(0: R^{\prime \prime} M^{\prime \prime}\right)=0$. Clearly, $\left|M^{\prime}\right|<\infty$ since $M^{\prime}$ is f.g. over the finite ring $R^{\prime}$. In the same way, $M^{\prime \prime}$ is f.g. over $R^{\prime \prime}$. Now an $R^{\prime \prime}$-submodule $N$ of $M^{\prime \prime}$ gives an $R$-submodule of $M$ by the one-to-one function $N \mapsto M^{\prime} \times N$. It follows that $M^{\prime \prime}$ is an FMS $R^{\prime \prime}$-module. Therefore, we can assume that $R$ is an FMIR with $|R / P|=\infty$ for each $P \in \operatorname{Spec}(R)=\left\{P_{1}, \ldots, P_{n}\right\}$. By Corollary 2.7, $M$ is generated over $R$ by some $e_{1}, \ldots, e_{n} \in M$ such that $M_{P_{i}}=R_{P_{i}}\left(e_{i} / 1\right)$ for each $i$. Actually, $e_{i} / 1$ is free over $R_{P_{i}}$ : suppose that $(a / t)\left(e_{i} / 1\right)=0$ for $a \in R$ and $t \in R \backslash P_{i}$. There is some $s_{i} \in R \backslash P_{i}$ such that $s_{i} a e_{i}=0$. Moreover, $e_{j} / 1 \in M_{P_{i}}=R_{P_{i}}\left(e_{i} / 1\right)$ for $j \neq i$ gives that $e_{j} / 1=\left(b_{j} / t_{j}\right)\left(e_{i} / 1\right)$, for some $b_{j} \in R, t_{j} \in R \backslash P_{i}$ for each $j \neq i$. This allows us to pick up some $s_{j} \in R \backslash P_{i}$ such that $s_{j} a e_{j}=0$. Setting $s:=s_{1} \cdots s_{n}$, we get $s a e_{k}=0$ for each $k \in\{1, \ldots, n\}$. Since $M$ is faithful, $s a=0$, so that $a / t=0$. By [2, Théorème 2 , ch.II, p.141], $M$ is a rank one projective $R$-module and (2) follows.

Conversely, assume that (1) and (2) hold and keep the above notation with $R=R^{\prime} \times R^{\prime \prime},\left|R^{\prime}\right|<\infty,\left|R^{\prime \prime} / P\right|=\infty$ for any $P \in \operatorname{Spec}\left(R^{\prime \prime}\right)$ and $M=M^{\prime} \times M^{\prime \prime}$, where $M^{\prime}$ is a finite $R^{\prime}$-module and $M^{\prime \prime}$ is a rank one projective $R^{\prime \prime}$-module. Then, from [2, Théorème 2, ch. II, p. 141], we deduce that $M^{\prime \prime}$ is f.g. over $R^{\prime \prime}$, with $M_{P}^{\prime \prime}$ cyclic for each maximal ideal $P$ of $R^{\prime \prime}$. Since $M^{\prime}$ is also f.g. over $R^{\prime}$ because finite, $M$ is f.g. over $R$. For each $N \in \operatorname{Max}(R)$ such that $|R / N|=\infty$, there exists $P \in \operatorname{Max}\left(R^{\prime \prime}\right)$ such that $N=R^{\prime} \times P$ and in this case $M_{N} \cong M_{P}^{\prime \prime}$ as $R_{N}$-modules. Indeed, consider the $R_{N}$-linear isomorphism $u: M_{N} \cong\left(M^{\prime} \times M^{\prime \prime}\right)_{R^{\prime} \times P} \rightarrow M_{P}^{\prime \prime}$ defined by $u\left(\left(m^{\prime}, m^{\prime \prime}\right) /(s, t)\right)=m^{\prime \prime} / t$, using the ring isomorphism $R_{N} \cong R_{P}^{\prime \prime}$. It 
follows that $M_{N}$ is cyclic over $R_{N}$. By Corollary 2.7, we can conclude that $M$ is an FMS module.

Remark 2.14. (1) For the proof of Theorem 2.13, it was convenient to suppose that $M$ is a faithful $R$-module. However, one should note that Theorem 2.13 can be used to characterize when an arbitrary (not necessarily faithful) module is FMS. In fact, an R-module $M$ is FMS (as an $R$-module) if and only if $M$ is an FMS module over the ring $R /(0: M)$.

(2) The rings $R^{\prime}$ and $R^{\prime \prime}$ in the statement of Theorem 2.13 are necessarily each FMIRs. In fact, if $A$ and $B$ are rings, then $A \times B$ is an FMIR if and only if both $A$ and $B$ are FMIRs.

Acknowledgments. The authors wish to thank the anonymous referees for making valuable suggestions for the improvement of this paper.

\section{References}

[1] D. D. Anderson and S. Chun, Commutative rings with finitely generated monoids of fractional ideals, J. Algebra, 320(7) (2008), 3006-3021.

[2] N. Bourbaki, Algèbre Commutative, Chs. 1 and 2, Hermann, Paris, 1961.

[3] N. Bourbaki, Algèbre, Chs. 1-3, Hermann, Paris, 1970.

[4] N. Bourbaki, Algèbre, Chs. 4-7, Masson, Paris, 1981.

[5] P.-J. Cahen, D. E. Dobbs and T. G. Lucas, Characterizing minimal ring extensions, Rocky Mountain J. Math., 41(4) (2011), 1081-1125.

[6] D. E. Dobbs, Every commutative ring has a minimal ring extension, Comm. Algebra, 34(10) (2006), 3875-3881.

[7] D. E. Dobbs, B. Mullins, G. Picavet and M. Picavet-L'Hermitte, On the FIP property for extensions of commutative rings, Comm. Algebra, 33(9) (2005), 3091-3119.

[8] D. E. Dobbs, G. Picavet and M. Picavet-L'Hermitte, Characterizing the ring extensions that satisfy FIP or FCP, J. Algebra, 371 (2012), 391-429.

[9] D. E. Dobbs and J. Shapiro, A classification of the minimal ring extensions of certain commutative rings, J. Algebra, 308 (2007), 800-821.

[10] D. Ferrand and J.-P. Olivier, Homomorphisms minimaux d'anneaux, J. Algebra, 16 (1970), 461-471.

[11] M. S. Gilbert, Extensions of Commutative Rings with Linearly Ordered Intermediate Rings, Ph. D. Dissertation, University of Tennessee, Knoxville, 1996.

[12] R. Gilmer, Some finiteness conditions on the set of overrings of an integral domain, Proc. Amer. Math. Soc., 131(8) (2003), 2337-2346. 
[13] J. A. Huckaba, Commutative Rings with Zero Divisors, Monographs and Textbooks in Pure and Applied Mathematics, 117, Marcel Dekker, Inc., New York, 1988.

[14] J. A. Huckaba and I. J. Papick, A note on a class of extensions, Rend. Circ. Mat. Palermo, 38 (1989), 430-436.

[15] D. G. Northcott, Lessons on Rings, Modules and Multiplicities, Cambridge University Press, London, 1968.

[16] G. Picavet and M. Picavet-L'Hermitte, About minimal morphisms, Multiplicative Ideal Theory in Commutative Algebra, Springer-Verlag, New York, 2006, 369-386.

[17] G. Picavet and M. Picavet-L'Hermitte, FIP and FCP products of ring morphisms, submitted.

[18] R. G. Swan, On seminormality, J. Algebra, 67 (1980), 210-229.

\section{Gabriel Picavet and Martine Picavet-L'Hermitte}

Université Blaise Pascal

Laboratoire de Mathématiques UMR 6620 CNRS

Les Cézeaux, 24 Avenue des Landais, BP 80026

63177 Aubière Cedex, France

e-mails: Gabriel.Picavet@math.univ-bpclermont.fr, picavet.gm@wanadoo.fr (G. Picavet)

Martine.Picavet@math.univ-bpclermont.fr, picavet.gm@wanadoo.fr (M. Picavet-L'Hermitte) 